\title{
A comparison of Bayesian and non-linear regression methods for robust estimation of pharmacokinetics in DCE-MRI and how it affects cancer diagnosis
}

\author{
Nikolaos Dikaios $^{\text {a) }}$, David Atkinson ${ }^{\text {b) }}$, Chiara Tudisca ${ }^{\text {b) }}$, Pierpaolo Purpura ${ }^{\text {b) }}$, Martin Forster ${ }^{\text {c,d) }}$, \\ Hashim Ahmed ${ }^{\mathrm{e})}$, Timothy Beale ${ }^{\mathrm{c})}$, Mark Emberton ${ }^{\mathrm{e})}$, and Shonit Punwani ${ }^{\mathrm{b}, \mathrm{c}}$ \\ a) Centre for Vision, Speech and Signal Processing, University of Surrey, UK \\ b) Centre for Medical Imaging, University College London, UK \\ ${ }^{c)}$ Department of Head and Neck Oncology, University College London Hospital, UK. \\ d) Cancer Institute, University College London, UK \\ e) Department of Urology, University College London, London, UK. NW1 2PG.
}

Author to whom correspondence should be addressed;

Dr Nikolaos Dikaios

Centre for Vision, Speech and Signal Processing

388 Stag Hill

Guildford

GU2 7XH

electronic mail: nd301@ @otmail.co.uk. 


\section{AbSTRACT}

The aim of this work is to compare Bayesian Inference for nonlinear models with commonly used traditional non-linear regression (NR) algorithms for estimating tracer kinetics in Dynamic Contrast Enhanced Magnetic Resonance Imaging (DCE-MRI). The algorithms are compared in terms of accuracy, and reproducibility under different initialization settings. Further it is investigated how a more robust estimation of tracer kinetics affects cancer diagnosis. The derived tracer kinetics from the Bayesian algorithm were validated against traditional NR algorithms (i.e. Levenberg-Marquardt, simplex) in terms of accuracy on a digital DCE phantom and in terms of goodness-of-fit (Kolmogorov-Smirnov test) on ROI-based concentration time courses from two different patient cohorts. The first cohort consisted of 76 men, 20 of whom had significant peripheral zone prostate cancer (any cancer-core-length $(\mathrm{CCL})$ with Gleason $>3+3$ or any-grade with $C C L>=4 \mathrm{~mm}$ ) following transperineal template prostate mapping biopsy. The second cohort consisted of 9 healthy volunteers and 24 patients with head and neck squamous cell carcinoma. The diagnostic ability of the derived tracer kinetics was assessed with receiver operating characteristic area under curve (ROC AUC) analysis. The Bayesian algorithm accurately recovered the ground-truth tracer kinetics for the digital DCE phantom consistently improving the Structural Similarity Index (SSIM) across the 50 different initializations compared to NR. For optimized initialization, Bayesian did not improve significantly the fitting accuracy on both patient cohorts, and it only significantly improved the $\mathrm{v}_{\mathrm{e}}$ ROC AUC on the HN population from ROC AUC $=0.56$ for the simplex to ROC $\mathrm{AUC}=0.76$. For both cohorts, the values and the diagnostic ability of tracer kinetic parameters estimated with the Bayesian algorithm weren't affected by their initialization. To conclude, the Bayesian algorithm led to a more accurate and reproducible quantification of tracer kinetic parameters in DCE-MRI, improving their ROC-AUC and decreasing their dependence on initialization settings.

Keywords: DCE analysis, Bayesian Inference for nonlinear model, Prostate cancer, Head and Neck 


\section{Acknowledgements}

This work was undertaken at the Centre for Vision, Speech and Signal Processing, University of Surrey and UCLH/UCL. This work was supported by UK EPSRC; Grant numbers: EP/I018700/1, EP/H046410/1 and the joint CR-UK \& EPSRC funded King's College London and UCL Comprehensive Cancer Imaging Centre, in association with the MRC and DoH (England). 


\section{Introduction}

Dynamic contrast enhanced (DCE) magnetic resonance imaging (MRI) is influenced by the micro-vascular characteristics of tissue, such as blood flow/volume, surface area/permeability of vessel walls, and micro-vascular density. These characteristics are associated with the expression of potent cytokines (such as the vascular endothelial growth factor) that support the development of tumor vessels. This makes DCE-MRI a valuable diagnostic tool in oncology. The purpose of this study is to investigate whether accurate quantification of tracer kinetic parameters using the proposed Bayesian Inference for nonlinear models can improve cancer diagnosis compared to traditional non-linear regression fitting algorithms.

Quantification of tracer kinetic parameters is affected by field inhomogeneities, gradients, SNR of the reconstructed images, and spatiotemporal resolution [1]. Besides limitations in acquisition, quantification of tracer kinetic parameters will depend on the selection of tracer kinetic model, the accurate estimation of the arterial input function, the estimation of the native $\mathrm{T} 1$ of the tissue [2] and the selection of fitting algorithm. Heyes et al. $[3,4]$ studied the variation within- and between workstations in the derivation of tracer kinetic parameters and reported a $25.1 \%-74.1 \%$ within-subject coefficient of variation. The conclusion of these studies is that unless the contrast agent material, the definition of AIF, the image SNR, and the fitting process are standardized DCE MRI related parameters will not be reproducible. Tracer kinetic models such as the extended Toft model [5] that describe the enhancement process are often used to derive quantitative parameters and are increasingly used in diagnostic models [6] including computer aided diagnostic (CAD) software [7, 8]. Accurate quantification that will be reproducible between different clinical sites is necessary for the widespread of DCE based CAD software. This work will investigate how the optimization process itself can affect the quantification and the diagnostic ability of the quantified parameters.

Quantitative DCE parameters are usually extracted by fitting the estimated concentration to the measured concentration time intensity course (TIC), using algorithms such 
as non-linear least squares or the simplex algorithm. These fitting algorithms are prone to hit local minima [9] resulting in fitting errors and fitted parameters that depend on their initialization. To the best of our knowledge there are no guidelines on how to initialize the tracer kinetics, and its clinical site uses its own initialization settings. Consequently there is a clear need to develop robust fitting strategies that will not be affected by the initialization of the tracer kinetics.

To overcome these issues, Bayesian inference for nonlinear models were suggested [10, 11, 12]. Bayesian algorithms can model the noise of the measured concentration of the contrast agent and have a theoretical guarantee to converge if run long enough [13]. This work suggests a Bayesian inference for nonlinear model algorithm similar to the ones proposed by other groups $[10,11,12]$ and evaluates its robustness and diagnostic value against the LevenbergMarquardt and the simplex algorithms on two separate cohorts of patients:

i) a cohort of 76 men, 20 of whom had significant prostate cancer in the peripheral zone

ii) a cohort of 9 healthy volunteers and 24 patients with squamous cell carcinoma.

The proposed Bayesian inference for nonlinear model algorithm is described in the theory section. The robustness value is assessed based on goodness-of-fit, and how robust the algorithm is when using different initialization settings of the estimated tracer kinetic parameters. Receiver operating characteristic (ROC) analysis is performed on the derived tracer kinetics to assess their ability to classify significant cancer.

\section{Theory}

\subsection{Tracer kinetic Modeling}

A tracer kinetic analysis was performed by fitting the extended Toft [5] (Eq. 1) modelled concentration $C(\mathrm{t})(\mathrm{mmol} / \mathrm{L})$ to the concentration time course $C_{T I C}(\mathrm{t}) \quad(\mathrm{Eq} .2)$.

$$
C(\mathrm{t})=v_{p} \cdot C_{a}(\mathrm{t})+K_{\text {trans }} \cdot \int_{0}^{\mathrm{t}} C_{a}\left(\tau-t_{0}\right) \otimes \mathrm{e}^{\left(-\frac{K_{\text {trans }}}{v_{e}}(t-\tau)\right)} \mathrm{d} \tau
$$


Where $C_{a}(\mathrm{t})$ is the arterial input function $(\mathrm{mmol} / \mathrm{L}), v_{p}$ is the blood plasma volume fraction, $K_{\text {trans }}$ is the transfer constant between plasma and interstitial space $\left(\min ^{-1}\right), v_{e}$ is the interstitial space volume and $t_{0}$ is the arrival time of the bolus at the tissue (secs). A population arterial input function was used [15].

The concentration time course was calculated from the image signal intensities $S(\mathrm{t})$ using the approximation Repetition time $\ll \mathrm{T} 1$

$$
C_{T I C}(\mathrm{t})=\frac{1}{r_{1} \cdot T 10}\left(\frac{S(\mathrm{t})-S_{0}}{S_{0}}\right)
$$

Where $r_{1}$ is the in-vivo relaxivity $\left(4.51 \mathrm{~L} \mathrm{mmol}^{-1} \mathrm{sec}^{-1}\right), T 10$ is the native $\mathrm{T} 1$ of the tissue before contrast agent injection, calculated from a multiple flip angle dataset (sec), and $S_{0}$ is the average of the acquired images before the injection of the contrast agent.

\subsection{Traditional nonlinear regression algorithms}

Tracer kinetic models are fitted with two commonly used non-linear regression algorithms i.e. the Levenberg-Marquardt and the simplex algorithm. Levenberg-Marquardt is a least squares curve fitting algorithm that is a blend between the Gauss-Newton and the gradient descent method. The update rule of the tracer kinetics parameters is: $k^{i+1}=k^{\mathrm{i}}-(\mathrm{H}+\lambda \mathrm{I})^{-1} \nabla L\left(k^{\mathrm{i}}\right)$, where $\mathrm{H}$ is the Hessian matrix at $k^{\mathrm{i}}, \lambda$ is a regularization parameter and $L$ is the likelihood function to be minimized i.e. $L\left(k^{\mathrm{i}}\right)=\sum_{t}\left(C_{T I C}(\mathrm{t})-C(\mathrm{t})\right)^{2}$. When the likelihood is decreased $\lambda$ is also reduced, but if the likelihood is increased $\lambda$ will also be increased to reduce the influence of gradient descent. Contrary to other gradient based methods Levenberg-Marquardt is not performing a line minimization (where the direction of gradient descent is decided prior to step size estimation) hence requires less likelihood evaluations reducing the computational cost. 
The simplex algorithm is also an iterative procedure but unlike the Levenberg-Marquardt does not require derivative information. The algorithm will create a "random" simplex of $n+1$ points, where $\mathrm{n}$ is the number dimensions (number of tracer kinetic parameters to be estimated). The simplex moves iteratively by reflection, expansion or contraction steps trying to find the tracer kinetic parameters that minimize the likelihood function. In this work we used a constrained variation of the simplex algorithm $[16,17]$ and a $\ell 1$-norm in the likelihood function to improve robustness [18]. The simplex algorithm is particularly advantageous in cases where the gradient of the likelihood functions is hard to calculate.

\subsection{Bayesian inference for nonlinear models}

In the proposed Bayesian Inference for nonlinear model algorithm the measured concentration $C_{T I C}(\mathrm{t})$ is modelled using additive Gaussian noise $\sigma^{2}$ and the tracer kinetic parameters, $k=\left\{\mathrm{v}_{\mathrm{p}}, \mathrm{K}_{\text {trans }}, \mathrm{v}_{\mathrm{e}}, \mathrm{t}_{0}\right\}$ for the extended Tofts model.

$$
C_{T I C}(\mathrm{t}) \sim \operatorname{normal}(C(\mathrm{t}), \sigma)
$$

The suggested Bayesian algorithm similar to $[10,11,12]$ maximizes the posterior probability distribution function $\mathrm{p}\left(\mathrm{k}, \sigma \mid \mathrm{C}_{\mathrm{TIC}}\right)$ as a function of $k$ and $\sigma$

$$
\hat{k}, \hat{\sigma}=\operatorname{argmax}_{k, \sigma} p\left(k, \sigma \mid C_{T I C}\right)
$$

According to the Bayes theorem $\mathrm{p}\left(\mathrm{k}, \sigma \mid \mathrm{C}_{\mathrm{TIC}}\right)$ is given by,

$$
p\left(k, \sigma \mid C_{T I C}\right)=p(k, \sigma) \cdot p\left(C_{T I C} \mid k, \sigma\right) \int_{k^{*}, \sigma^{*}} p\left(k^{*}, \sigma^{*}\right) \cdot p\left(C_{T I C} \mid k^{*}, \sigma^{*}\right)
$$

Where $p\left(C_{T I C} \mid k, \sigma\right)$ is the likelihood function of $C_{T I C}$ given the tracer kinetic parameters $k$,

$$
p\left(C_{T I C} \mid k, \sigma\right)=\left(2 \pi \sigma^{2}\right)^{-1} \exp \left(-\frac{1}{2 \sigma^{2}}\left\|C_{T I C}(\boldsymbol{r}, t)-C(\boldsymbol{r}, t)\right\|_{2}^{2}\right)
$$

and $\mathrm{p}(k, \sigma)$ is the product of the prior probability distribution functions of $\mathrm{k}$ and $\sigma, p(\mathrm{k}, \sigma)$.

Prior probability distribution functions reflect our prior knowledge about the $k$ and $\sigma^{2}$ parameters. Similarly to Schmid et al. [10] the subsequent prior distributions are assumed for every tracer kinetic parameter:

- $\quad \mathrm{v}_{\mathrm{p}}$ follows a Beta distribution, $\mathrm{v}_{\mathrm{p}} \sim \boldsymbol{B e t a}(1,19)[19]$ reflecting an a priori expected value of 0.05 . 
- $K_{\text {trans }}$ was parameterized as suggested by Schmid et al. [10] as $\mathrm{e}^{\theta}$ where $\theta$ follows a Gaussian distribution $\theta \sim \operatorname{Normal}(0,1)$

- $\mathrm{v}_{\mathrm{e}}$ follows a Beta distribution, $\mathrm{v}_{\mathrm{e}} \sim$ Beta $(2,1.5)$ reflecting an a priori expected value of 0.57

- $\mathrm{t}_{0}$ follows a uniform random distribution unif (injection time, injection time+40).

- $\sigma^{2}$ follows an uninformative Inverse Gamma distribution IG $\left(10^{-4}, 10^{-4}\right)$

The integral $\int_{k^{*}, \sigma^{*}} p\left(k^{*}, \sigma^{*}\right) \cdot p\left(y \mid k^{*}, \sigma^{*}\right)$ is estimated with the Metropolis-Hastings algorithm.

\section{Materials and methods}

\subsection{Generate Simulated DCE data}

The DCE simulation used is similar to the one published from our group in Dikaios et al. (2014) [20]. A normal volunteer underwent a fast gradient echo DCE-MRI protocol (flip angle $\alpha=10^{\circ}$, repetition time TR $=2.3$ msecs). A T1-weighted abdominal image was acquired without contrast injection. The first time-frame was manually segmented into: liver, bowel, right and left heart, aorta, portal vein. Such segmentation was used as a map to simulate contrast enhancement using the extended Tofts model or the dual input function Orton model for the liver. Ground truth parametric maps i.e. native T10 (range 382-1932 msecs), $v_{p}\left(\right.$ range 0-1), $v_{e}$ (range 0-1), and $\mathrm{K}_{\text {trans }}\left(\right.$ range $0-1.38 \mathrm{~min}^{-1}$ ) were used to simulate fifty DCE images with temporal resolution 3 secs using the spoiled gradient echo model.

$$
S(t)=\rho \frac{\sin (\alpha) \cdot\left(1-\exp \left(-\frac{T R}{T 1(t ; k)}\right)\right)}{1-\cos (\alpha) \cdot \exp \left(-\frac{T R}{T 1(t ; k)}\right)}
$$

Where $\rho$ is the proton density image, and was calculated analytically using Eq. 8 from the T1weighted abdominal image without contrast injection and the graund truth T10 maps.

DCE images were transformed to $(k, t)$-space with fast Fourier transformation where noise was added. The noise of complex valued $(\mathrm{k}, \mathrm{t})$-space MR data can be reasonably modelled by an additive white Gaussian distribution on both real and imaginary components (independent and identically distributed random variables). Simulated DCE data were generated for 2 different 
noise levels, one corresponding to the average SNR before contrast injection of prostate T1w images (SNR 9.2, noise level=2500) and a separate one corresponding to the average SNR before contrast injection of neck T1w images (SNR 15.1, noise level=800). The SNRs were calculated as described in Dikaios et al. [21].

\subsection{Patient populations}

Institutional review board (IRB) approval for the study was obtained. The requirement for consent was waived for use of images acquired in routine clinical practice (prostate peripheral zone population) and obtained from all patients undergoing imaging as part of a separate clinical trial (head and neck population).

\section{Prostate population}

The prostate population consisted of men with clinically suspected prostate cancer (elevated prostate specific antigen (PSA) \pm abnormal digital rectal examination \pm family history of prostate cancer \pm urinary symptoms,) undergoing prostatic multiparametric MRI (mp-MRI: T2 weighted, diffusion weighted and DCE imaging) prior to template-prostate-mapping (TPM) biopsies as part of standard of care at our institution. In total 76 men (mean age 63 years, range $45-79)$ with a mean prostate specific antigen of $7.8 \mathrm{ng} / \mathrm{ml}(\mathrm{range} 1.2-20 \mathrm{ng} / \mathrm{ml}$ ) and a mean prostate gland volume of $48.2 \mathrm{ml}$ (range $23-137 \mathrm{ml}$ ) were included from 06/2007 to 03/2011. Twenty of the 76 men had histologically verified clinically significant peripheral zone prostate cancer.

Imaging was performed using a 1.5T magnet (Avanto, Siemens, Erlangen, Germany) with a pelvic phased array coil. The contrast media was Dotarem with an application dose 0.2 $\mathrm{mL} /$ Kgr. Prior to imaging, $0.2 \mathrm{mg} / \mathrm{kg}$ (maximum $20 \mathrm{mg}$ ) of spasmolytic (Buscopan; Boehringer Ingelheim, Ingelheim, Germany) was administered intravenously to reduce peristalsis. DCEMRI was performed with a T1 weighted volumetric FLASH sequence with TR/TE 5.61/2.5 ms, flip angle $15^{\circ}, 384 \times 384$ matrix dimensions, field of view $269 \mathrm{~mm}$, slice thickness $3 \mathrm{~mm}, 26$ 
reconstructed slices, temporal resolution of 16 seconds, and number of time points 35 .

For the purpose of this study and to match with the target performance of mp-MRI as defined by recent consensus [24]; histopathologists identified all locations with clinically significant cancer based on volume assessment $(0.2 \mathrm{ml})$ estimated by the cancer core length $(\mathrm{CCL})>=4 \mathrm{~mm}$ and/or the presence of Gleason pattern 4 disease [25]. Small volume $(<0.2 \mathrm{ml})$ and low grade $(<=$ Gleason $3+3)$ tumour was identified as clinically insignificant cancer.

An experienced radiologist (with 10 years of mp-MRI experience, reporting $500 \mathrm{mp}-$ MRI prostate scans/year) and using the TPM biopsy histology as a guide, carefully matched the histopathology template to the mp-MRI; and contoured a region of interest (ROI) on early contrast enhanced T1 weighted images at the single largest histologically confirmed significant cancer site. For patients where the entire prostate was benign or contained only insignificant cancer, the radiologist contoured a $1-\mathrm{cm}^{2} \mathrm{ROI}$ at a confirmed benign location within the PZ.

\section{Head and Neck population}

Twenty-four consecutive patients (mean age 60 years, standard deviation 9 years, range 44 to 80 years) satisfying inclusion criteria of histologically confirmed head and neck SCC with cervical nodal metastatic disease at pre-therapy staging, and 9 normal volunteers (mean age 48 years, standard deviation 16 years, range 20 to 75 years) were recruited between March 2010 and May 2012. All patients underwent contrast enhanced neck computed tomography (CECT), anatomical MRI and neck ultrasound as part of routine pre-treatment staging; and were consented for additional DCE MRI of the neck for research purposes.

All MRI studies were acquired using a 1.5T Siemens Avanto (Siemens, Erlangen, Germany) magnet with the manufacturer's carotid coils. The contrast media was Dotarem with an application dose $0.2 \mathrm{~mL} / \mathrm{Kgr}$. DCE-MRI was performed with a T1 weighted volumetric FLASH sequence with TR/TE 2.3/1.0 ms, flip angle $10^{\circ}, 256 \times 256$ matrix dimensions, field of view 269 $\mathrm{mm}$, slice thickness $4 \mathrm{~mm}$, temporal resolution of 3 seconds, and number of time points 50 . 
The reference standard was established by experienced head and neck radiologists (with 8 years and 24 years of head and neck experience respectively) through review of all CT and anatomical MRI, and performance of ultrasound evaluation of the neck in all patients. Cervical nodes were assessed as per the Union for International Cancer Control (UICC): Tumor nodal-metastasis (TNM) classification of malignant tumours [26]. Equivocal nodes were sampled at the time of ultrasound by fine needle aspiration (FNA) and classified by in-room cytology.

\subsection{Optimization details of the fitting algorithms}

Fitting algorithms were implemented with in-house-developed software in MATLAB (The Mathworks Inc, Natick, MA). The tracer kinetic parameters for the simplex, the Levenberg-Marquardt and the Bayesian inference for nonlinear model were initialized $\operatorname{as~}_{\mathrm{p}}{ }^{0}=$ $0.05, \mathrm{~K}_{\text {trans }}{ }^{0}=0.4 \mathrm{~min}^{-1}, \mathrm{v}_{\mathrm{e}}^{0}=0.5$ for both the simplex and the Levenberg-Marquardt. The constraints of the tracer kinetic parameters were: $v_{p} \in[0,1], K_{\text {trans }} \in[0,2.7] \mathrm{min}^{-1}, v_{e} \in$ $[0,1], t_{0} \in[$ injection time, injection time +40 secs $]$. Onset time was initialized with the time point the contrast agent was administered.

The simplex, the Levenberg-Marquardt and the Bayesian inference for nonlinear model were run using multiple initialisations of the tracer kinetic parameters. In addition to the aforementioned initialization, 49 different initialisations were also generated (50 initializations in total) using uniform distributions supported within intervals as described by the following formulas: $\mathrm{v}_{\mathrm{p}}^{0}=$ unif $(0,0.2), \mathrm{K}_{\text {trans }}^{0}=$ unif $(0.3,1.0), \mathrm{v}_{\mathrm{e}}^{0}=$ unif $(0.3,0.6)$.

For the proposed Bayesian inference for nonlinear model the total number of iterations was 500 , burn-in iterations were 300 , thinning equal to 5 , and tune iteration (number of iterations for tuning) was 67.

\subsection{Statistical analysis}

Statistical analysis was performed using SPSS (SPSS Base 20.0 for Windows. SPSS 
Inc., Chicago IL). The same statistical analysis was performed for both the head and neck and PZ prostate population.

A Mann-Whitney U test (MWU sig) was performed to compare the median values of the tracer kinetic parameters between normal and cancer ROIs. The goodness-of-fits were assessed with the Kolmogorov-Smirnov (KS) test statistic.

Separate univariate logistic regression models were built for the tracer kinetic parameters derived using the simplex and the Bayesian algorithms. The ability of individual tracer kinetic parameters to classify cancer was assessed by receiver operator characteristic (ROC) area under curve (AUC) analysis.

Leave-one-out analysis [21] was used for internal validation of predictive models. One case (out of the total patient population) was excluded, and a model generated from the remainder of the cases. The model was then tested on the excluded case and a predictive probability calculated. The process was repeated for all cases, excluding successive cases in turn allowing calculation of a predictive probability per case. An ROC (LOO ROC) was then created using the derived predictive probabilities. ROC curves were compared using the significance test suggested by Hanley and McNeil [27].

\section{Results}

\subsection{Simulated DCE data}

Table 1 demonstrates the similarity in terms of Structural SIMilarity (SSIM) index of the estimated tracer kinetic maps estimated with the simplex, the Levenberg Marquardt and the Bayesian inference for nonlinear model to the ground truth tracer kinetic maps. Results are shown for two different noise realizations, one corresponding to the SNR of prostate $\mathrm{T} 1 \mathrm{w}$ images ( 9.2) before contrast injection and one corresponding to the SNR of neck T1w images ( 15) before contrast injection. The tracer kinetic maps estimated with the Bayesian algorithm have substantially higher SSIM and are less affected from the different initializations of the 
tracer kinetic parameters (lower interquartile range across the 50 different initializations). The simplex algorithm has similar performance to the Levenberg-Marquardt (LM), with marginally higher SSIM. Fig. 1 provides a visual comparison between the tracer kinetics maps estimated with the simplex and the Bayesian algorithm.

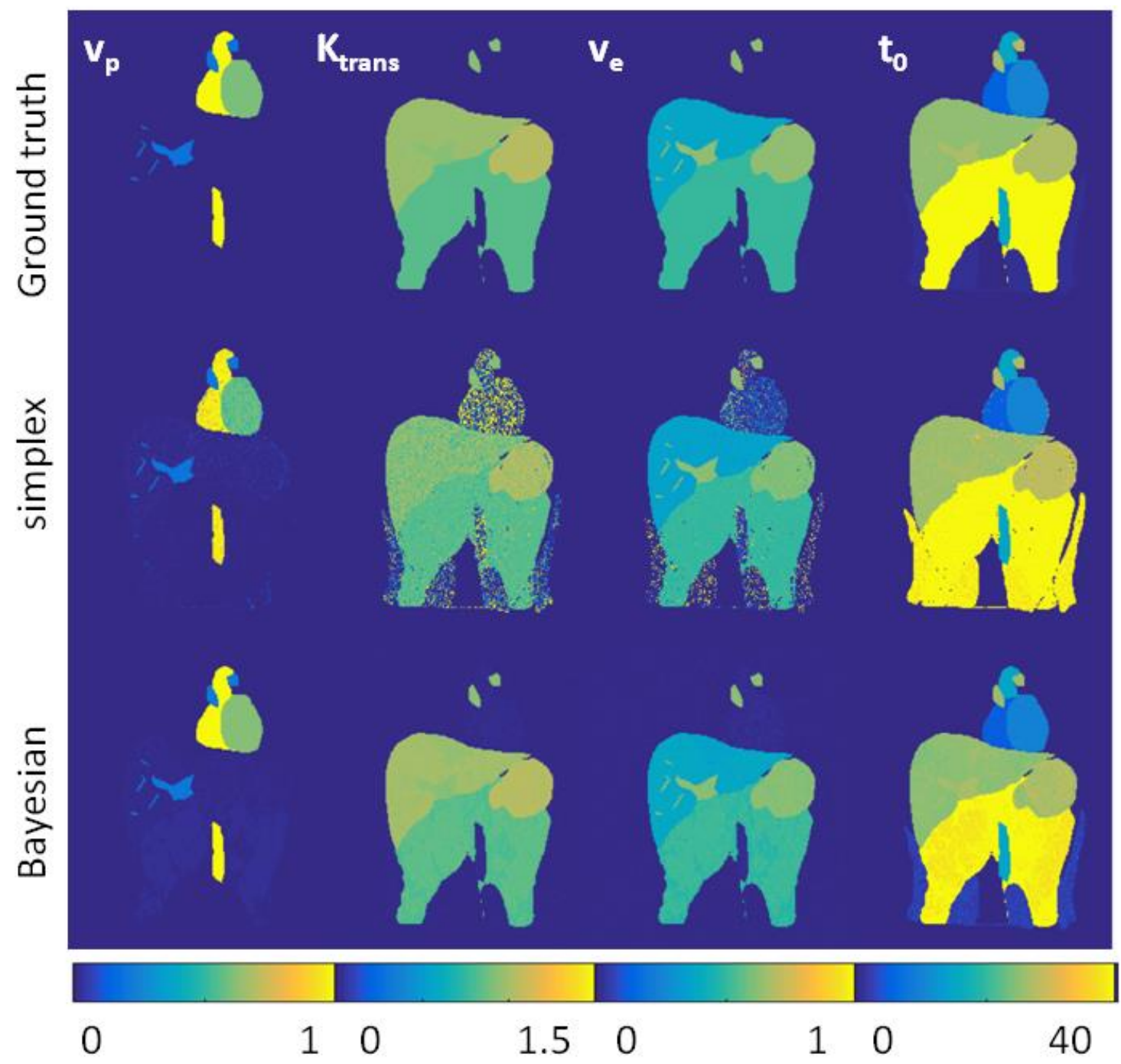

Fig. 1

Parametric maps $\left(\mathrm{v}_{\mathrm{p}}, \mathrm{K}_{\text {trans }}, \mathrm{v}_{\mathrm{e}}, \mathrm{t}_{0}\right.$ ) estimated with pixel-by-pixel fitting of the simulated DCE images with SNR=9.2 using the simplex and the Bayesian inference for nonlinear model. Ground truth tracer kinetics maps are shown at the top row.

\section{Table 1}

SIMilarity (SSIM) index between the parametric maps $\left(\mathrm{v}_{\mathrm{p}}, \mathrm{K}_{\text {trans }}, \mathrm{v}_{\mathrm{e}}, \mathrm{t} 0\right)$ estimated with pixel-by- 
pixel fitting (using the simplex, the Levenberg Marquardt and the Bayesian algorithms) and the ground truth parametric maps of simulated DCE data. Results are shown for different noise realizations with $\mathrm{SNR}=9.2$ (prostate T1w images) and $\mathrm{SNR}=15$ (neck T1w images). Median and Interquartile range (iQR) of SSIM were calculated across the 50 different initializations for each method.

\begin{tabular}{|c|c|c|c|c|}
\hline Median (iQR) & $\mathrm{v}_{\mathrm{p}}$ & $\mathrm{K}_{\text {trans }}$ & $\mathrm{V}_{\mathrm{e}}$ & $\mathrm{t}_{0}$ \\
\hline \multicolumn{5}{|c|}{ SNR=9.2 } \\
\hline simplex & $0.90(0.17)$ & $0.69(0.13)$ & $0.81(0.14)$ & $0.70(0.12)$ \\
\hline LM & $0.89(0.17)$ & $0.69(0.14)$ & $0.80(0.15)$ & $0.68(0.14)$ \\
\hline Bayesian & $0.92(0.02)$ & $0.81(0.01)$ & $0.84(0.02)$ & $0.76(0.01)$ \\
\hline \multicolumn{5}{|c|}{$\mathrm{SNR}=15$} \\
\hline simplex & $0.95(0.11)$ & $0.81(0.10)$ & $0.87(0.08)$ & $0.79(0.09)$ \\
\hline LM & $0.95(0.11)$ & $0.81(0.10)$ & $0.86(0.08)$ & $0.79(0.09)$ \\
\hline Bayesian & $0.98(<0.01)$ & $0.91(<0.01)$ & $0.93(0.01)$ & $0.86(0.01)$ \\
\hline
\end{tabular}

\subsection{Prostate population}

\section{Multiple initialisations - Robustness of evaluated algorithms}

The simplex, the Levenberg-Marquardt and the Bayesian algorithm were all run with the same 50 different initializations, Fig. 2 shows the KS test statistic (across the 76 mean ROI TICs of the PZ prostate population) for each initialization. The interquartile range of the medians was 0.019 for the simplex algorithm, 0.018 for the Levenberg-Marquardt algorithm and 0.002 for the Bayesian algorithm. The simplex algorithm had consistently better goodness-of-fit than the Levenberg-Marquardt; hence hereafter the Levenberg-Marquardt was excluded from the comparison. 


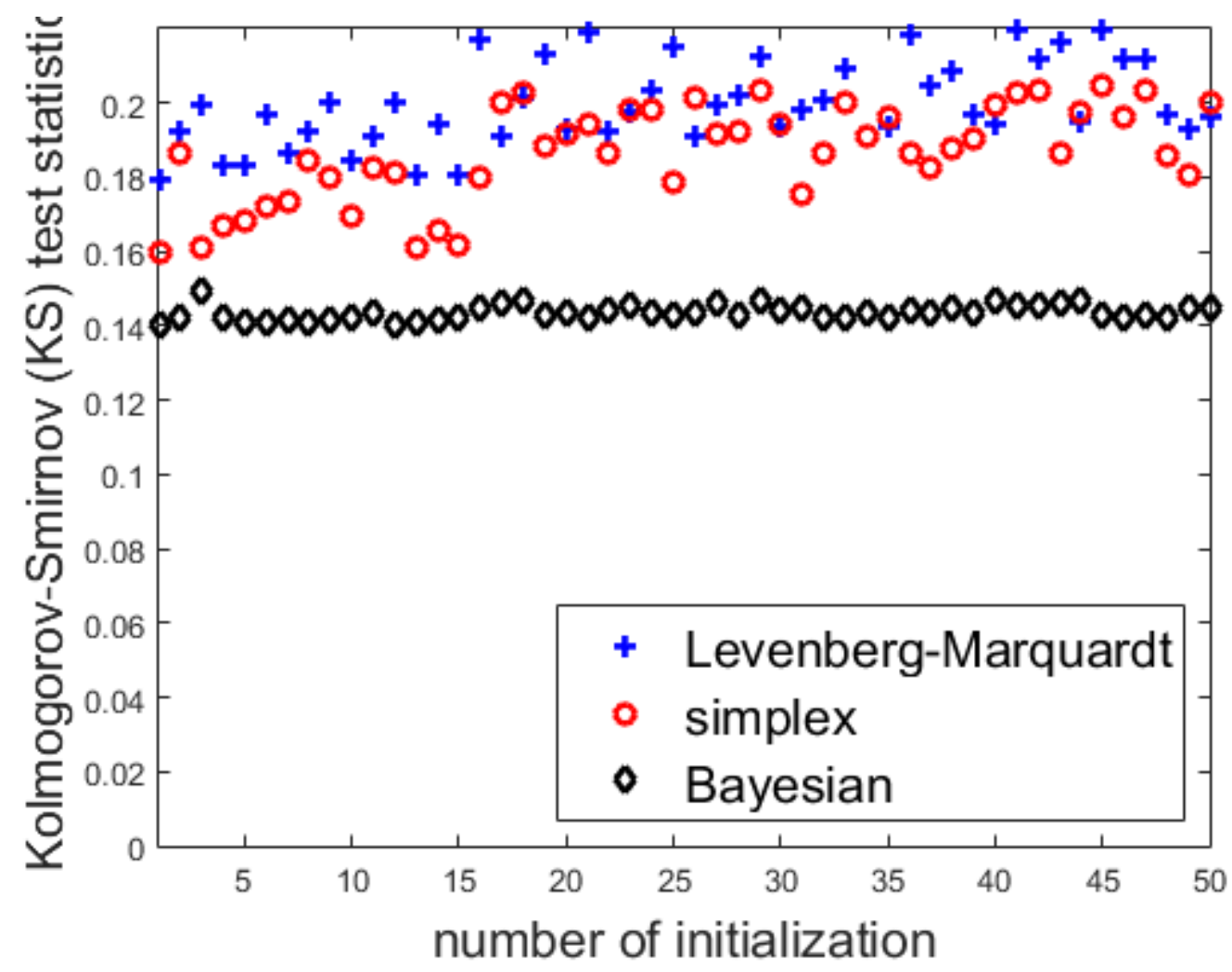

Fig. 2

Plot of the median KS statistic test (median KS statistic test across the 76 mean ROI PZ prostate TICs) across the 50 different initializations for the Levenberg-Marquardt, the simplex and the Bayesian algorithms. The interquartile range (iQR) of the median KS statistic test is 0.019 for the Levenberg-Marquardt, 0.018 for the simplex and 0.002 for the Bayesian.

\section{Univariate ROC analysis}

Table 2 shows the ROC analysis of the tracer kinetic parameters estimated with the simplex and the Bayesian algorithms using the optimum tracer kinetic initialization in terms of goodness-offit. $\mathrm{K}_{\text {trans }}$ was the best classifier of PZ prostate cancer for both the simplex and the Bayesian algorithm. According to the score test only $\mathrm{K}_{\text {trans }}$ estimated with the Bayesian algorithm could significantly discriminate PZ prostate cancer $(\mathrm{p}=0.02)$ (Table 2). However following a significance test between ROC curves, the AUC of $\mathrm{K}_{\text {trans }}$ estimated with the Bayesian algorithm (shown in Table 2) was not significantly better.

The simplex and the Bayesian algorithms were run with different initializations as 
described in section 3.3 and the ROC AUC were estimated per tracer kinetic parameter for each initialization. The median (interquartile range) ROC AUC across the 50 different initializations were $\mathrm{v}_{\mathrm{p}}: 0.55(0.05), \mathrm{K}_{\text {trans }}: 0.57(0.14)$, and $\mathrm{v}_{\mathrm{e}}: 0.56(0.05)$ for the simplex algorithm and $\mathrm{v}_{\mathrm{p}}: 0.63$ (0.02), $\mathrm{K}_{\text {trans: }}: 0.67$ (0.02), and $\mathrm{v}_{\mathrm{e}}: 0.56$ (0.01) for the Bayesian algorithm. The median ROC AUC values for $\mathrm{v}_{\mathrm{p}}$ and $\mathrm{K}_{\text {trans }}$ between the simplex and the Bayesian were significantly different.

\section{Table 2}

Score test and univariate ROC analysis of the tracer kinetic parameters derived with the simplex and the Bayesian algorithms (using the optimum tracer kinetic initialization in terms of goodnessof-fit) performed on the ROI-based TIC from the whole PZ population and following LOO analysis.

\begin{tabular}{llccc}
\hline & & score (p-value) & ROC AUC (CI) & LOO ROC AUC (CI) \\
\hline \hline \multirow{2}{*}{ simplex } & $\mathrm{v}_{\mathrm{p}}$ & $0.21(0.65)$ & $0.61(0.47-0.76)$ & $0.22(0.11-0.33)$ \\
& $\mathrm{K}_{\text {trans }}$ & $3.22(0.07)$ & $0.64(0.50-0.78)$ & $0.57(0.41-0.72)$ \\
& $\mathrm{v}_{\mathrm{e}}$ & $0.69(0.41)$ & $0.54(0.40-0.68)$ & $0.41(0.28-0.54)$ \\
& & & & \\
& $\mathrm{v}_{\mathrm{p}}$ & $2.31(0.13)$ & $0.58(0.43-0.74)$ & $0.48(0.32-0.65)$ \\
Bayesian & $\mathrm{K}_{\text {trans }}$ & $5.46(0.02)$ & $0.67(0.54-0.81)$ & $0.63(0.50-0.77)$ \\
& $\mathrm{v}_{\mathrm{e}}$ & $0.75(0.39)$ & $0.56(0.41-0.71)$ & $0.44(0.30-0.58)$ \\
\hline
\end{tabular}

\section{Comparison of tracer kinetic parameters between $\mathrm{PZ}$ prostate cancer/benign ROIs}

Parametric maps of a PZ prostate cancer patient estimated with the simplex and the Bayesian algorithms are illustrated in Fig. 3. The modelled concentration $\mathrm{C}(\mathrm{t})$ is fitted to the mean concentration TIC along the $\mathrm{PZ}$ prostate cancer ROI $\mathrm{C}_{\mathrm{TIC}}(\mathrm{t})$ (Fig. 3). In Fig. 3, while $\mathrm{v}_{\mathrm{p}}$ values estimated from the cancer ROI TIC are almost zero for the simplex algorithm, following pixelby-pixel fitting the cancer area in the $\mathrm{v}_{\mathrm{p}}$ seems to be slightly higher than zero. Tracer kinetic parameters estimated by fitting mean ROI TICs will not necessarily correlate with tracer kinetic parameters estimated by pixel-by-pixel fitting. Taking the mean of an ROI and propagating it in time will generate a "smooth" profile, resulting in an approximated time-intensity curve. Ideally 
pixel-by-pixel fitting needs to be performed, but because it is more computationally demanding many clinical papers resort to mean ROI TIC fitting.

Following MWU test, none of the tracer kinetic parameters estimated with the Bayesian algorithm were significantly different from the ones estimated with the simplex algorithm for either the benign or the cancer ROIs (Fig. 4).

Early enhancement
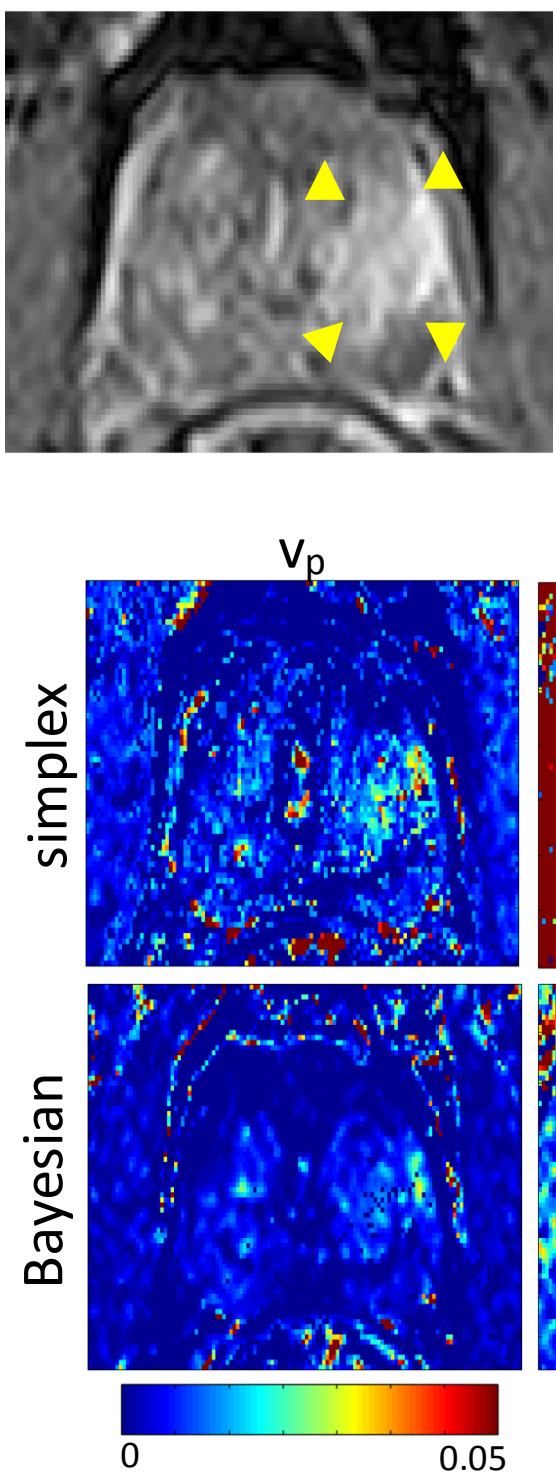
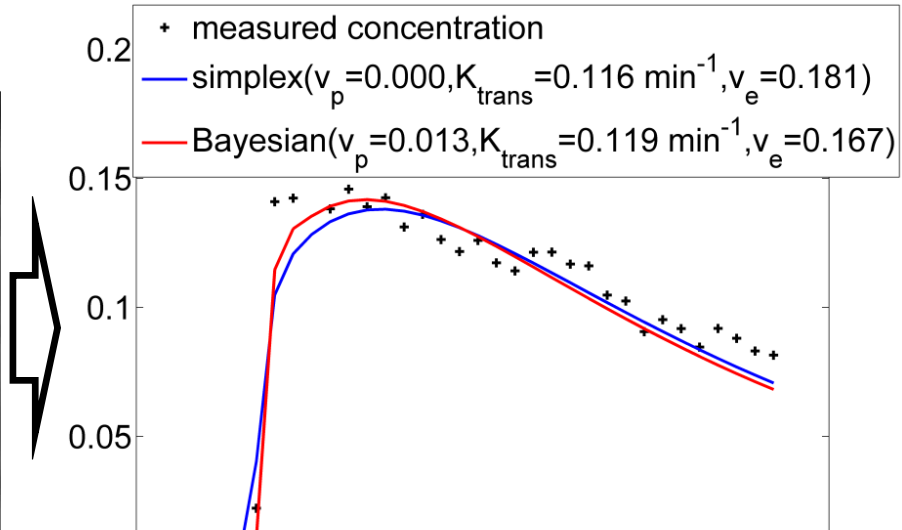

0.15
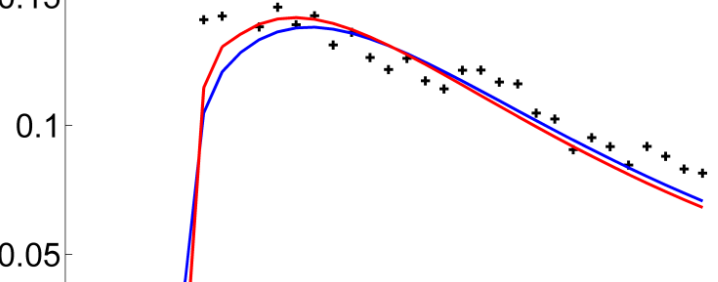
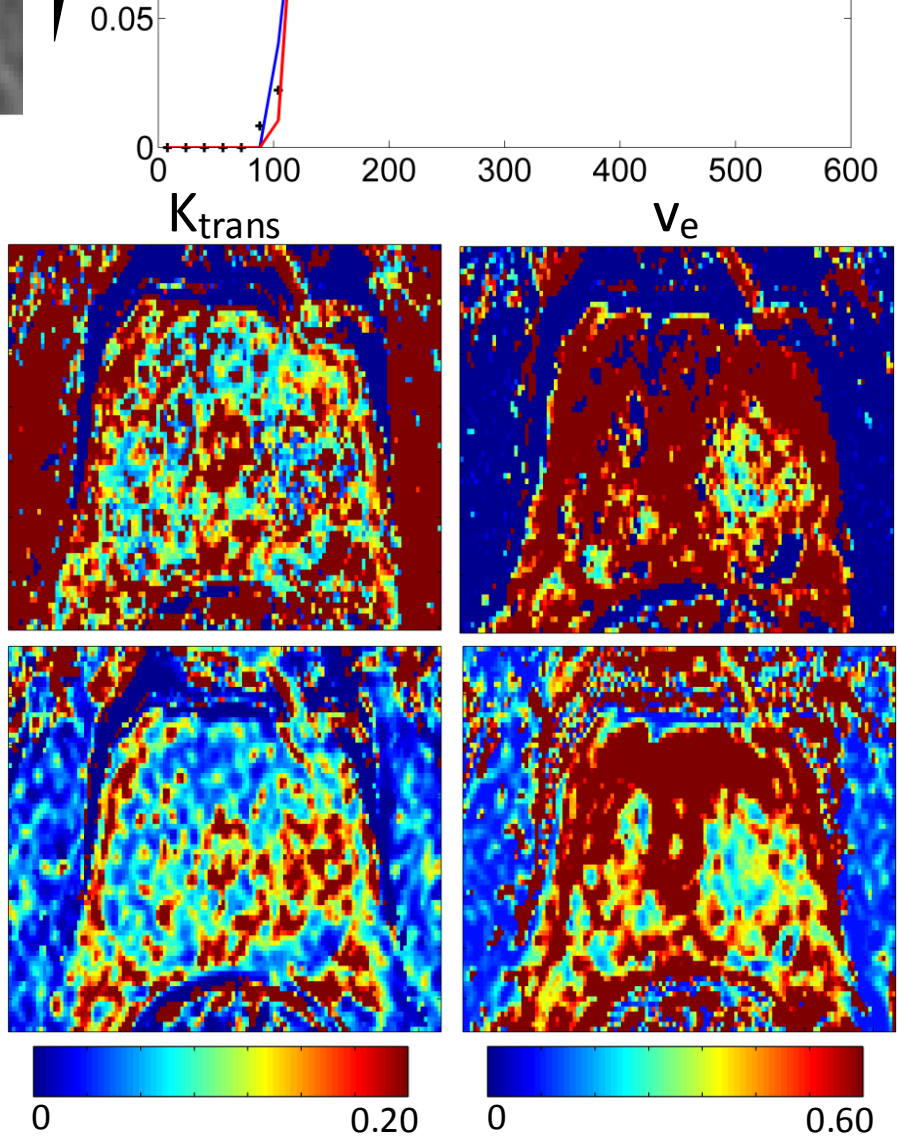

Fig. 3

Tracer kinetic maps $\left(\mathrm{v}_{\mathrm{p}}, \mathrm{K}_{\text {trans }}, \mathrm{v}_{\mathrm{e}}\right)$ estimated with pixel-by-pixel fitting using the simplex and the Bayesian algorithms for a PZ prostate cancer patient. A plot of the mean ROI concentration TIC $C_{T I C}(\mathrm{t})$ and the fitted to curve using the simplex and the Bayesian algorithms is also shown. 

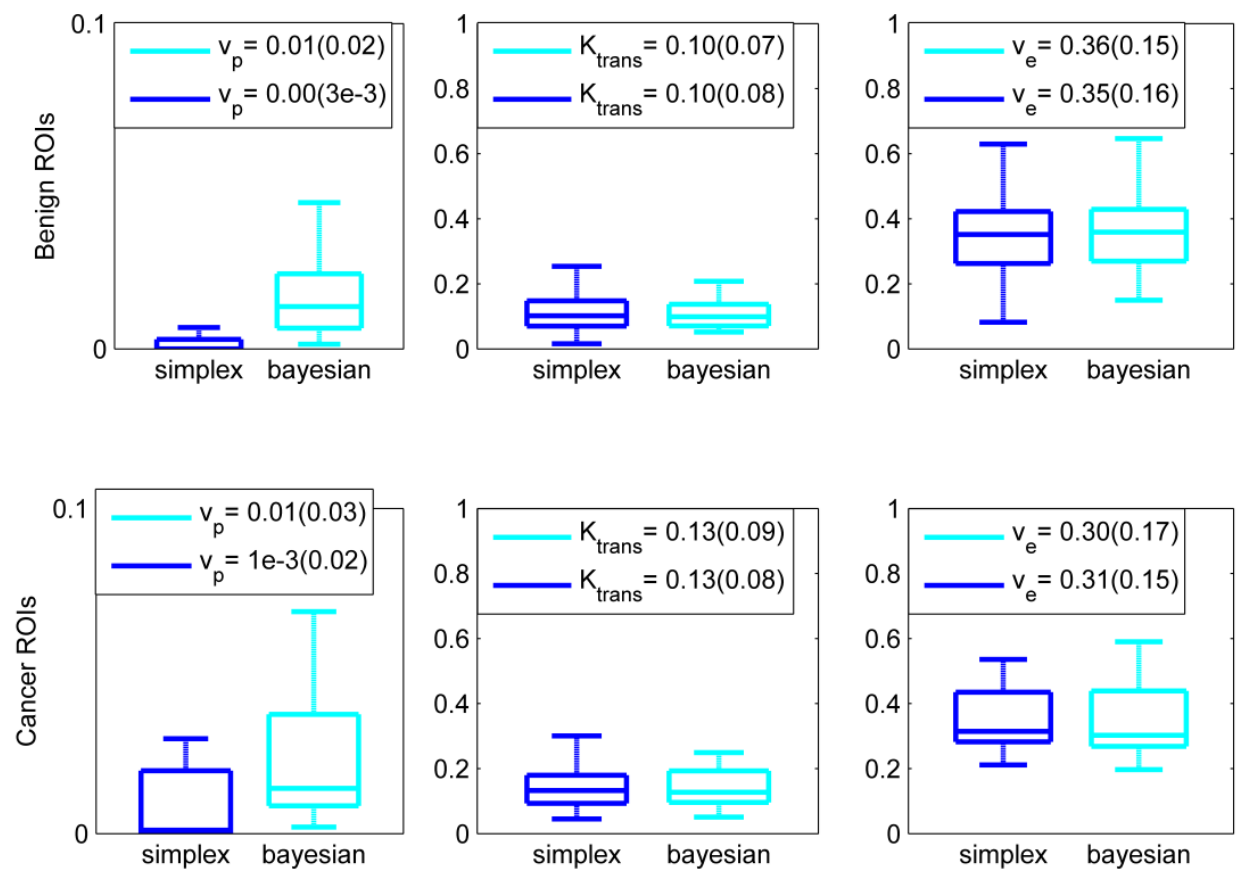

\section{Fig. 4}

Boxplot diagram of the tracer kinetic parameters derived with the simplex and the proposed Bayesian algorithm, performed separately for the normal and cancer PZ prostate ROI based TIC. The terms in brackets refer to the median value (interquartile range) of the estimated tracer kinetic parameters.

\subsection{Head and Neck population}

\section{Multiple initialisations - Robustness of evaluated algorithms}

The simplex, the Levenberg-Marquardt and the Bayesian algorithms were all run with the same 50 different initializations, Fig. 5 shows the median KS statistic test (across the 33 mean ROI TICs of the head and neck patients and volunteers) for each initialization. The interquartile range of the medians was 0.0083 for the simplex algorithm, 0.010 for the Levenberg-Marquardt algorithm and 0.0021 for the Bayesian algorithm. Simplex algorithm had consistently better goodness-of-fit than the Levenberg-Marquardt; hence from hereafter the Levenberg-Marquardt was excluded from the comparison. 


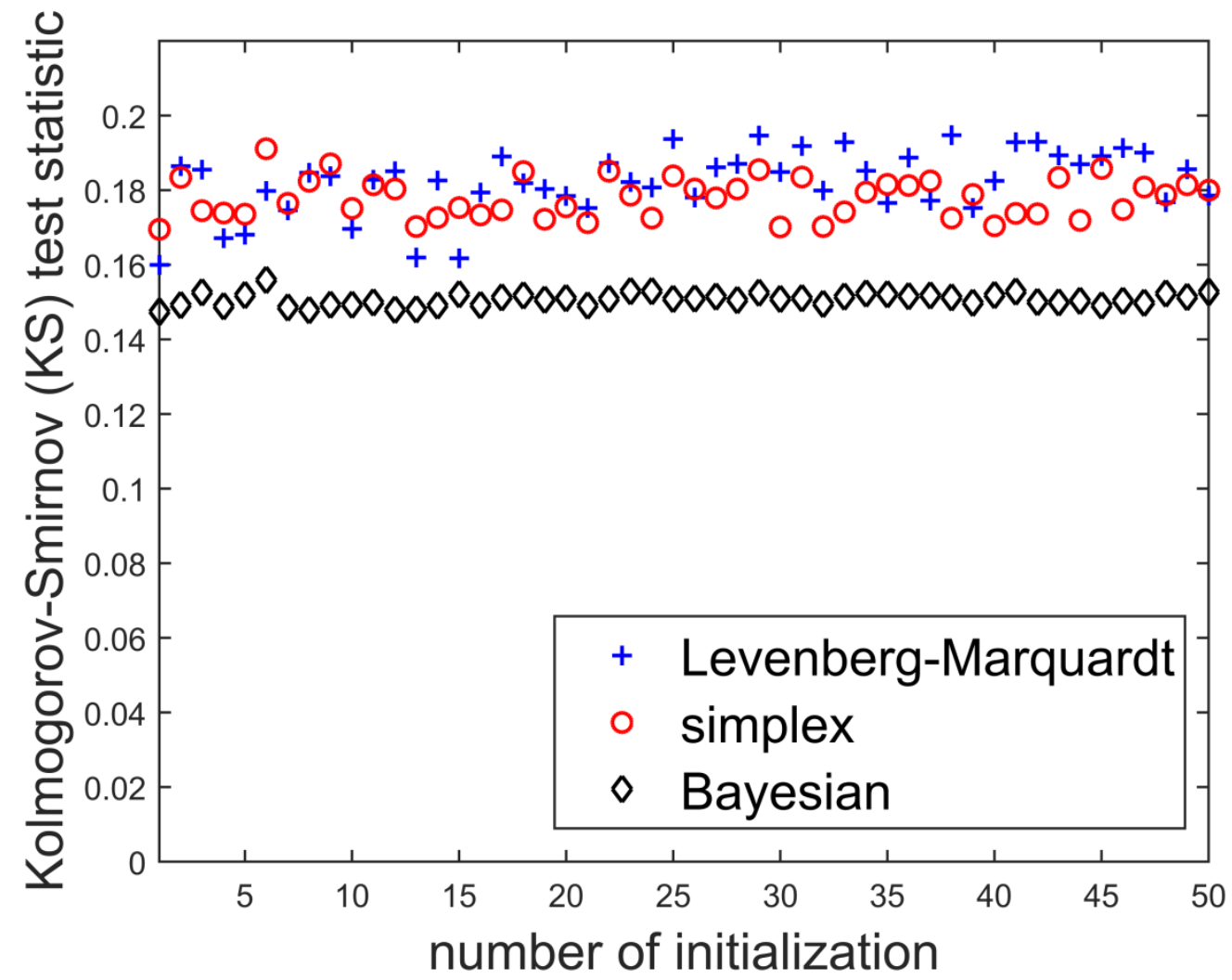

Fig. 5

Plot of the median KS statistic test (median KS statistic test across the 33 mean ROI head and neck TICs) across the 50 different initializations for the simplex and the Bayesian algorithms. The interquartile range (iQR) of the median KS statistic test is 0.010 for the LevenbergMarquardt, 0.0083 for the simplex and 0.0021 for the Bayesian algorithm.

\section{Univariate ROC analysis}

Table 3 shows the ROC analysis of the tracer kinetic parameters estimated with the simplex and the Bayesian algorithms using the optimum tracer kinetic initialization in terms of goodness-of-fit. $\mathrm{K}_{\text {trans }}$ was the best classifier of head and neck metastatic patients for both the simplex and the Bayesian algorithms. According to the score test, for the simplex algorithm only $K_{\text {trans }}$ could significantly classify metastatic patients, whereas for the Bayesian both $K_{\text {trans }}$ and $v_{e}$ were significant classifiers (table 3 ).

Following a significance test between ROC curves, the AUC (on the original population or 
following LOO analysis) of $\mathrm{K}_{\text {trans, }}$, estimated with the Bayesian algorithm, was not significantly better. Significant difference was only found for the $v_{e}$ AUC between the simplex and the Bayesian algorithms (table 3).

The simplex and the Bayesian algorithms were run with different initializations as described in section 3.3 and the ROC AUC were estimated per tracer kinetic parameter for each initialization. ROC AUC were estimated per tracer kinetic parameter for each initialization. The median (interquartile range) ROC AUC across the 50 different initializations were $\mathrm{v}_{\mathrm{p}}$ : $0.54(0.14)$, $\mathrm{K}_{\text {trans }}: 0.76(0.13)$, and $\mathrm{v}_{\mathrm{e}}: 0.56(0.15)$ for the simplex algorithm and $\mathrm{v}_{\mathrm{p}}: 0.59(0.03), \mathrm{K}_{\text {trans }}: 0.81$ (0.01), and $\mathrm{v}_{\mathrm{e}}: 0.79(0.02)$ for the Bayesian algorithm. The median ROC AUC values for $\mathrm{v}_{\mathrm{e}}$ between the simplex and the Bayesian were significantly different.

\section{Table 3}

Score test and univariate ROC analysis of the tracer kinetic parameters derived with the simplex and the Bayesian algorithms (using the optimum tracer kinetic initialization in terms of goodnessof-fit) performed on the ROI-based TIC from the whole head and neck patient population and following LOO analysis. Asterisk (*) denotes the cases where the tracer kinetic parameter estimated with the Bayesian algorithm is significantly different from the corresponding one derived with the simplex algorithm.

\begin{tabular}{llccc}
\hline & & score (p-value) & ROC AUC $(\mathrm{CI})$ & LOO ROC AUC (CI) \\
\hline \hline \multirow{4}{*}{ simplex } & $\mathrm{v}_{\mathrm{p}}$ & $0.12(0.73)$ & $0.56(0.33-0.79)$ & $0.30(0.13-0.48)$ \\
& $\mathrm{K}_{\text {trans }}$ & $5.43(0.02)$ & $0.74(0.58-0.90)$ & $0.66(0.43-0.89)$ \\
& $\mathrm{v}_{\mathrm{e}}{ }^{*}$ & $0.54(0.49)$ & $0.56(0.34-0.77)$ & $0.31(0.15-0.50)$ \\
& & & & \\
& $\mathrm{v}_{\mathrm{p}}$ & $1.05(0.31)$ & $0.58(0.35-0.80)$ & $0.51(0.31-0.72)$ \\
Bayesian & $\mathrm{K}_{\text {trans }}$ & $6.37(0.01)$ & $0.80(0.64-0.94)$ & $0.75(0.57-0.92)$ \\
& $\mathrm{v}_{\mathrm{e}}{ }^{*}$ & $4.76(0.03)$ & $0.76(0.60-0.93)$ & $0.70(0.52-0.89)$ \\
\hline
\end{tabular}

\section{Comparison of tracer kinetic parameters between metastatic/benign ROIs}

Parametric maps of a head and neck metastatic patient estimated with the simplex and the 
Bayesian algorithms are illustrated in Fig. 6. Fitting the estimated concentration $\mathrm{C}(\mathrm{t})$ to the mean ROI concentration TIC along the head and neck metastatic nodes $\mathrm{C}_{\mathrm{TIC}}(\mathrm{t})$ is also shown in Fig. 6.

Following MWU test all the tracer kinetic parameters estimated with the Bayesian algorithm were significantly different from the ones estimated with the simplex algorithm for both the benign and the cancer ROIs (Fig. 7).
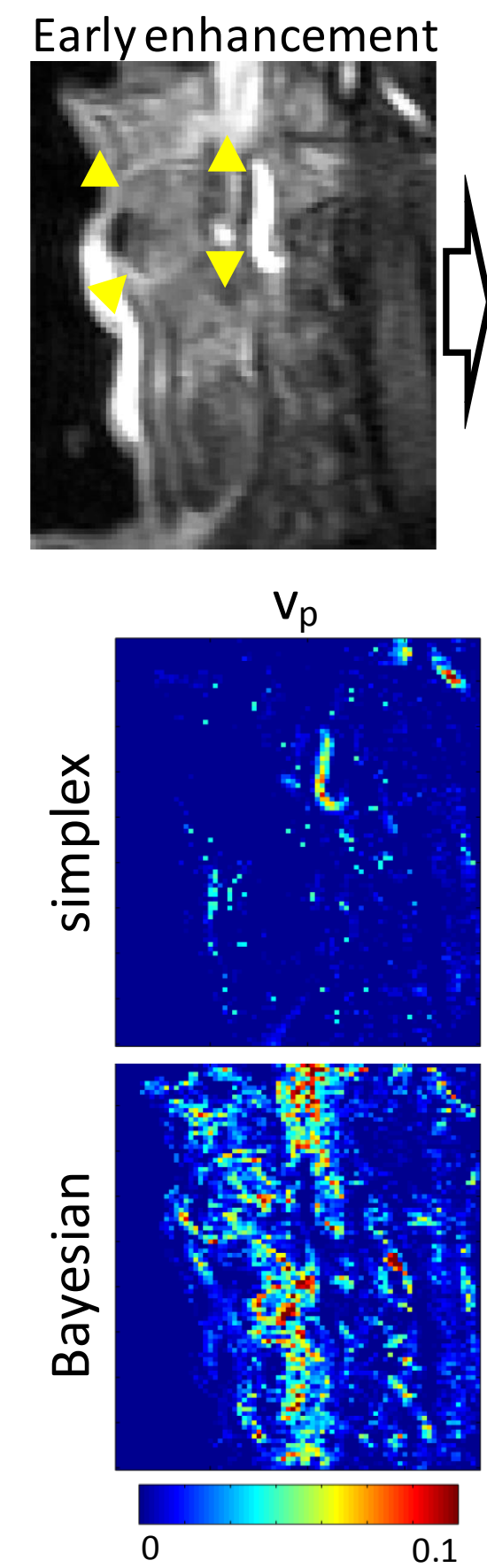
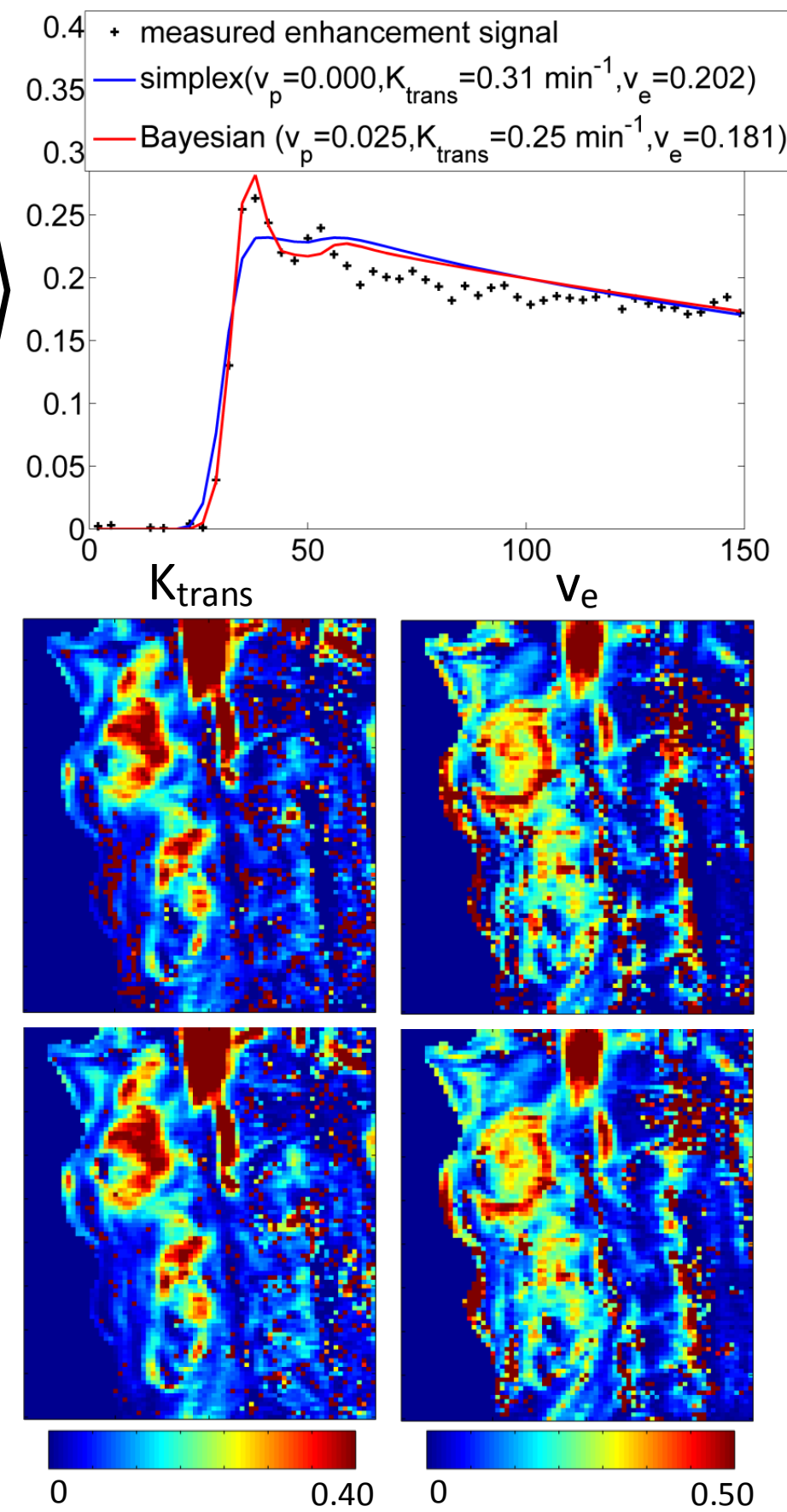

Fig. 6 
Parametric tracer kinetic maps $\left(\mathrm{v}_{\mathrm{p}}, \mathrm{K}_{\text {trans }}, \mathrm{v}_{\mathrm{e}}\right)$ estimated with pixel-by-pixel fitting using the simplex and the Bayesian algorithms for a head and neck patient with a metastasis. A plot of the mean ROI concentration TIC $C_{T I C}(\mathrm{t})$ and the fitted curve using the simplex and the Bayesian algorithms is also shown.
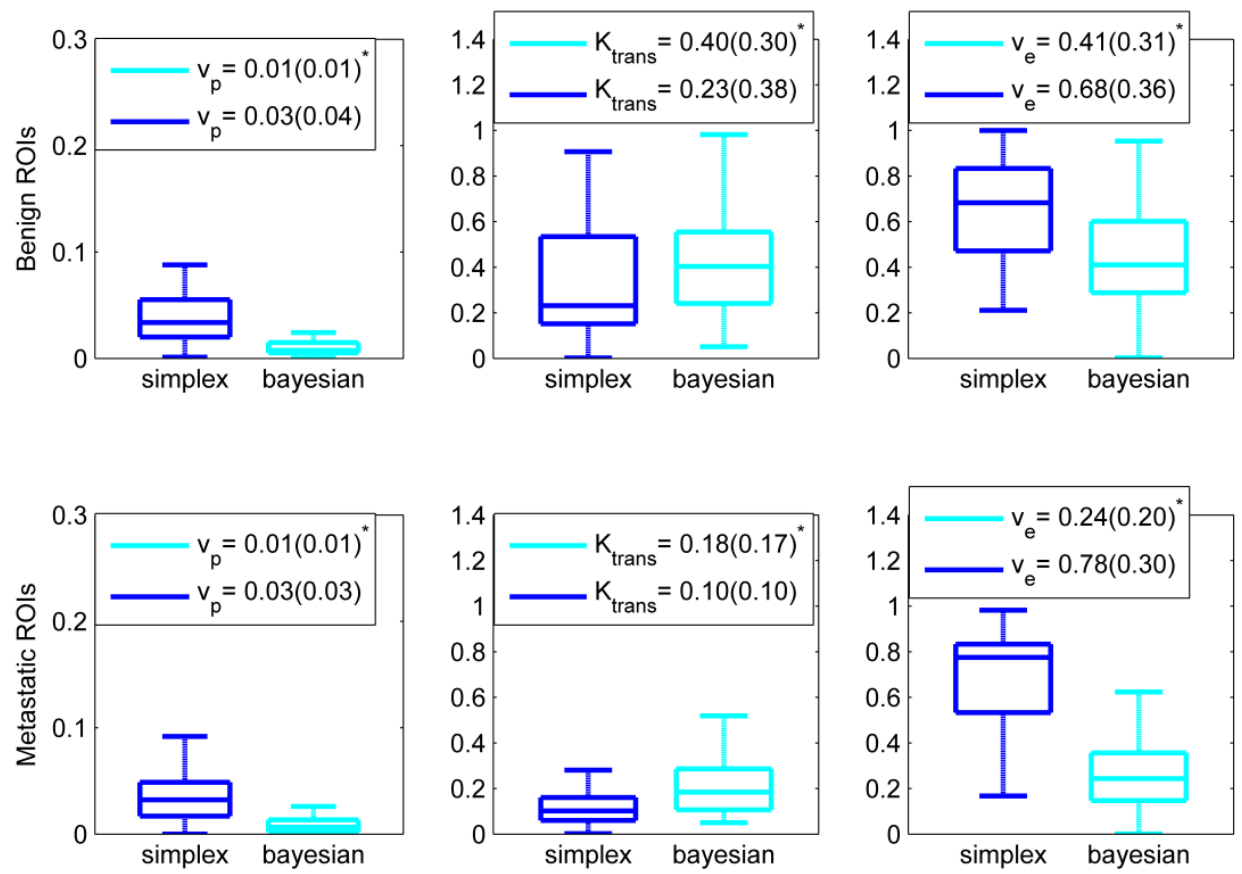

Fig. 7

Box-plot diagram of the tracer kinetic parameters derived with the simplex and the proposed Bayesian algorithms, performed separately for the benign and metastatic neck node ROIs. The terms in brackets refer to the median value (interquartile range) of the estimated tracer kinetic parameters. Asterisk $(*)$ denotes significant difference $(\mathrm{p}<0.05)$ between the simplex and Bayesian algorithms.

\section{Discussion}

This works aims to investigate the diagnostic benefits of using Bayesian algorithms for the derivation of tracer kinetic parameters in DCE-MRI. The proposed Bayesian algorithm is compared against traditional non-linear regression algorithms (i.e. Levenberg-Marquardt and 
simplex) in terms of accuracy, reproducibility under different initialization settings and ability to classify cancer.

The simplex algorithm had consistently marginally higher SSIM with the ground truth kinetics of the simulated DCE phantom and better goodness-of-fit for the ROI-based TIC of both populations than the Levenberg-Marquardt, which could be attributed to its convergence properties [17]. Unlike the Levenberg-Marquardt, the simplex algorithm does not use gradients, which provides some resilience to noise and local minima. Nonetheless both the LevenbergMarquardt and the simplex algorithm are prone to hit local minima, which is evident from our data. Re-initialization could reduce the risk to stop at a local minima. However to the best of our knowledge there is no optimum way on how to re-initialize the algorithms for DCE-MRI tracer kinetic quantification. Alternatively this work proposes a Bayesian inference for nonlinear model algorithm, which is shown to be resilient to local minima. Specifically, when running the proposed Bayesian for different initializations we found that

i. The SSIM with the ground truth tracer kinetic maps for the Bayesian algorithm was consistently higher than for the non-linear regression algorithms for all initializations.

ii. The goodness-of-fit (KS statistic test) for the Bayesian algorithm was almost constant and consistently lower than the non-linear regression algorithms for all initializations and for both populations.

iii. The ROC AUC of the tracer kinetic parameters estimated with the Bayesian algorithms have an interquartile range across the different initializations up to 0.03 , whereas for the simplex algorithm the interquartile range is up to 0.14 ( $\mathrm{PZ}$ prostate population) and 0.15 (head and neck population).

Tracer kinetic parameters estimated with the proposed Bayesian algorithm had higher classification ability for both PZ prostate and head and neck cancer. Tracer kinetic parameters estimated with the simplex algorithm that could not significantly classify disease, when estimated with the proposed Bayesian algorithm were significant classifiers of PZ prostate cancer (i.e. $\mathrm{K}_{\text {trans }}$ ) and metastatic head and neck cancer (i.e. $v_{\mathrm{e}}$ ). However the ROC AUC improvement achieved 
with the Bayesian algorithm was not significant for the PZ prostate cancer. For the head and neck metastasis only the ROC AUC improvement for $\mathrm{v}_{\mathrm{e}}$ was significant.

Bayesian inference for nonlinear model algorithms have been proposed before in the literature $[10,11,12]$ to estimate unbiased quantitative tracer kinetic parameters. The proposed scheme is similar to the one suggested by Schmid et al. [10], the main difference is on the estimation of the onset time. The accuracy of the estimated tracer kinetic parameters will depend on the arrival time of the contrast agent to the tissue (onset time) [28]. Schmid et al. [10] calculated the onset time as the minimum time $t^{*}$, for which the contrast concentration significantly exceeds zero minus $\mathrm{C}\left(\mathrm{t}^{*}\right) / \partial_{\mathrm{t}} \mathrm{C}\left(\mathrm{t}^{*}\right)$. For the simulated DCE phantom with $\mathrm{SNR}=9.2$, the SSIM index of the onset time calculated with the method of Schmid et al. [10] is 0.5754 , whereas for the proposed Bayesian algorithm the respective SSIM is 0.76 (Table 1). This affected the estimation of the tracer kinetic parameters, but if the same onset time was used the Bayesian method suggested by Schmid et al. [10] has similar performance with the one proposed in this work. This is expected since both use the Metropolis-Hastings Markov chain Monte Carlo (MCMC) method and similar prior information. Their only difference is that we parameterized the posterior probability distribution function $\mathrm{p}\left(\mathrm{k}, \sigma \mid \mathrm{C}_{\mathrm{TIC}}\right)$ with $\mathrm{v}_{\mathrm{e}}$ to optimize EES volume directly instead of calculating it via $\mathrm{k}_{\mathrm{ep}}\left(\mathrm{v}_{\mathrm{e}}=\mathrm{K}_{\text {trans }} / \mathrm{k}_{\mathrm{ep}}\right)[7]$.

\section{Limitations}

For the PZ prostate population, we were reliant upon visual matching of the Barzell zone histology on TPM with the ROIs on the mp-MRI. Therefore, results may be influenced by mis-registration errors. Although no biopsy is free from sampling error [29] we used TPM to address as much of the systematic error inherent to transrectal ultrasound (TRUS) guided biopsy as possible [29, 30]. For the head and neck population there was a relatively small sample size. We took great care to be certain about positive and negative disease status within individual nodes by recruiting patients 
with $\mathrm{N} 2 / 3$ disease confirmed by CT, MRI and US \pm FNA.

\section{Conclusions}

DCE MRI tracer kinetic parameters are increasingly used in clinical practice; their diagnostic ability will depend on their accurate and reproducible quantification. The proposed Bayesian inference for nonlinear model algorithm has been shown in this work to improve the diagnostic ability compared to the simplex algorithm and was robust when different initializations of the tracer kinetic parameters were used. These assets of the algorithm are essential to train and validate robust CAD software based on DCE-MRI that could be used between different sites. The performance of the Bayesian algorithm was consistent on two different populations, acquired with different settings.

\section{References}

[1] Yuan J, Chow SK, Yeung DK, Ahuja AT, King AD. Quantitative evaluation of dual-flip-angle T1 mapping on DCE-MRI kinetic parameter estimation in head and neck. Quant Imaging Med Surg. 2012; 2: 245-53.

[2] Heye T, Boll DT, Reiner CS, Bashir MR, Dale BM, Merkle EM. Impact of precontrast T10 relaxation times on dynamic contrast-enhanced MRI tracer kinetic parameters: T10 mapping versus a fixed T10 reference value. J Magn Reson Imaging. 2014; 39(5):1136-45.

[3] Heye T, Davenport MS, Horvath JJ, Feuerlein S, Breault SR, Bashir MR, Merkle EM, Boll DT. Reproducibility of dynamic contrast-enhanced MR imaging. Part I. Perfusion characteristics in the female pelvis by using multiple computer-aided diagnosis perfusion analysis solutions. Radiology. 2013; 266(3):801-11.

[4] Heye T, Merkle EM, Reiner CS, Davenport MS, Horvath JJ, Feuerlein S, Breault SR, Gall P, Bashir MR, Dale BM, Kiraly AP, Boll DT. Reproducibility of dynamic contrast-enhanced MR imaging. Part II. Comparison of intra- and interobserver variability with manual region of interest placement versus semiautomatic lesion segmentation and histogram analysis. Radiology. 2013; 266(3):812-21.

[5] Tofts PS. Modeling tracer kinetics in dynamic Gd-DTPA MR imaging. J Magn Reson Imaging 1997; 7(1):91-101.

[6] Dikaios N, Alkalbani J, Abd-Alazeez M, Sidhu HS, Kirkham A, Ahmed HU, Emberton M, 
Freeman A, Halligan S, Taylor S, Atkinson D, Punwani S. Zone-specific logistic regression models improve classification of prostate cancer on multi-parametric MRI. Eur Radiol. 2014; 25(9):2727-37.

[7] Sung YS, Kwon HJ, Park BW, Cho G, Lee CK, Cho KS, Kim JK. Prostate cancer detection on dynamic contrast-enhanced MRI: computer-aided diagnosis versus single perfusion parameter maps. AJR Am J Roentgenol. 2011; 197(5):1122-9.

[8] Kim MH, Kim JK, Lee Y, Park BW, Lee CK, Kim N, Cho G, Choi HJ, Cho KS. Diagnosis of lymph node metastasis in uterine cervical cancer: usefulness of computer-aided diagnosis with comprehensive evaluation of MR images and clinical findings. Acta Radiol. 2011; 52:1175-83.

[9] McKinnon, K.I.M. Convergence of the Nelder-Mead simplex method to a non-stationary point. SIAM J Optimization 1999; 9: 148-158.

[10] Schmid V, Witcher B, Padhani A, Taylor N and Yang G-Z. Bayesian methods for pharmacokinetic models in dynamic constrast-enhanced magnetic resonance imaging. IEEE Trans. Med. Imaging 2006; 25 1627-36.

[11] Bhushan M, Schnabel JA, Risser L, Heinrich MP, Brady JM, Jenkinson M. Motion correction and parameter estimation in dceMRI sequences: application to colorectal cancer. Med Image Comput Comput Assist Interv 2011; 14:476-83.

[12] Orton MR, Collins DJ, Walker-Samuel S, d'Arcy JA, Hawkes DJ, Atkinson D, Leach MO. Bayesian estimation of tracer kinetic parameters for DCE-MRI with a robust treatment of enhancement onset time. Phys Med Biol. 2007; 52(9):2393-408.

[13] Gelman A and Shirley K. Inference from simulations and monitoring convergence. In Handbook of Markov Chain Monte Carlo, ed.IS. Brooks, A. Gelman, G. Jones, and X. L. Meng. CRC Press, 2011.

[14] Orton MR, Miyazaki K, Koh DM, Collins DJ, Hawkes DJ, Atkinson D, Leach MO. Optimizing functional parameter accuracy for breath-hold DCE-MRI of liver tumours. Phys Med Biol. 2009; 54: 2197-215.

[15] Parker GJ, Roberts C, Macdonald A, Buonaccorsi GA, Cheung S, Buckley DL, Jackson A, Watson Y, Davies K, Jayson GC. Experimentally-derived functional form for a populationaveraged high-temporal-resolution arterial input function for dynamic contrast-enhanced MRI. Magnetic Resonance in Medicine 2006; 56: 993-1000.

[16] Errico JD, 2005. Bound Constrained Optimization, Computer Program. MATLAB and Simulink Centre, The MathWorks, Inc.

[17] Lagarias JC, Reeds JA, Wright MH, and Wright PE. Convergence Properties of the NelderMead Simplex Method in Low Dimensions. SIAM Journal of Optimization 1998; 9:112-147. [18] Press WH, Teukolsky SA, Vetterling WT, Flannery BP. Numerical Recipes in C. Cambridge, 
New York, 1994.

[19] Schmid VJ, Whitcher B, Padhani AR, Taylor NJ, Yang GZ. A Bayesian hierarchical model for the analysis of a longitudinal dynamic contrast-enhanced MRI oncology study. Magn Reson Med. 2008; 61(1):163-74.

[20] Dikaios N, Arridge S, Hamy V, Punwani S, Atkinson D. Direct parametric reconstruction from undersampled ( $k, t)$-space data in dynamic contrast enhanced MRI. Med Image Anal 2014; 18(7): 989-1001.

[21] Dikaios N, Punwani S, Hamy V, Purpura P, Rice S, Forster M, Mendes R, Taylor S, Atkinson D. Noise estimation from averaged diffusion weighted images: Can unbiased quantitative decay parameters assist cancer evaluation? Magn Reson Med. 2014; 71: 2105-17.

[22] Onik G, Barzell W. Transperineal 3D mapping biopsy of the prostate: an essential tool in selecting patients for focal prostate cancer therapy. Urol Oncol 2008; 26:506-10.

[23] Taira AV, Merrick GS, Galbreath RW, Andreini H, Taubenslag W, Curtis R, Butler WM, Adamovich E, Wallner KE. Performance of transperineal template-guided mapping biopsy in detecting prostate cancer in the initial and repeat biopsy setting. Prostate Cancer Prostatic Dis. $2010 ; 13: 71-7$.

[24] Dickinson L, Ahmed HU, Allen C, Barentsz JO, Carey B, Futterer JJ, Heijmink SW, Hoskin PJ, Kirkham A, Padhani AR, Persad R, Puech P, Punwani S, Sohaib AS, Tombal B, Villers A, van der Meulen J, Emberton M. Magnetic resonance imaging for the detection, localisation, and characterisation of prostate cancer: recommendations from a European consensus meeting. Eur Urol 2011; 59(4):477-94.

[25] Dikaios N, Alkalbani J, Sidhu HS, Fujiwara T, Abd-Alazeez M, Kirkham A, Allen C, Ahmed H, Emberton M, Freeman A, Halligan S, Taylor S, Atkinson D, Punwani S. Logistic regression model for diagnosis of transition zone prostate cancer on multi-parametric MRI. Eur Radiol. 2015; 25(2): 523-32.

[26] Sobin LH. TNM classification of malignant tumours. 7 ed. Chichester, West Sussex, UK; Hoboken, NJ: John Wiley and Sons, 2009.

[27] Hanley JA, McNeil BJ. The meaning and use of the area under a Receiver Operating Characteristic (ROC) curve. Radiology, 1982; 143, 29-36.

[28] Calamante F, Gadian D and Connelly A. Delay and dispersion effects in dynamic susceptibility contrast MRI: simulations using singular value decomposition. Magn. Reson. Med. 2000; 44: 466-73.

[29] Ahmed HU, Emberton M, Kepner G, Kepner J. A biomedical engineering approach to mitigate the errors of prostate biopsy. Nat Rev Urol. 2012; 9:227-31.

[30] Hoeks CM, Barentsz JO, Hambrock T, Yakar D, Somford DM, Heijmink SW, Scheenen 
TW, Vos PC, Huisman H, van Oort IM, Witjes JA, Heerschap A, Fütterer JJ. Prostate cancer: multiparametric MR imaging for detection, localization, and staging. Radiology 2011; 261:4666. 